\title{
ANÁLISE CLADÍSTICA DO GÊNERO Octobranchus MARION \& BOBRETZKY, 1875 (TRICHOBRANCHIDAE: POLYCHAETA)
}

\author{
GARRAFFONI, A.R.S. ${ }^{1,2,3} \&$ P.C. LANA ${ }^{1}$ \\ ${ }^{1}$ Centro de Estudos do Mar, Universidade Federal do Paraná, Av. Beira Mar s/n, Caixa \\ Postal 02, Pontal do Sul, CEP 83255-000, Pontal do Paraná, PR, Brasil. E-mail: \\ agarraffoni@lycos.com, lana@cem.ufpr.br \\ ${ }^{2}$ Curso de Pós-Graduação em Ciências Biológicas - Zoologia - Universidade Federal \\ do Paraná - Setor de Ciências Biológicas - Departamento de Zoologia, Caixa Postal \\ 19020 - CEP 81531-990 Curitiba - Paraná \\ ${ }^{3}$ Bolsista CNPq
}

\begin{abstract}
RESUMO
O gênero Octobranchus contém atualmente nove espécies válidas: Octobranchus pacificus (Berkeley \& Berkeley, 1954), O. antarcticus Monro, 1936, O. floriceps Kingston \& Mackie, 1980, O. japonicus Hessle, 1917, O. lingulatus (Grube, 1863), O. longipes Blankensteyn \& Lana, 1987, O. myunus Hutchings \& Peart, 2000, O. phyllocomus Hartman, 1952 e 0. sexlobatus Hartmann- Schröder \& Rosenfeldt, 1989 com ampla distribuição mundial (Mar do Norte, Mar do Japão, Australia, Brasil, Mediterrâneo e Antartica). O monofiletismo de Octobranchus é sustentado pela presença de quatro pares de brânquias, presentes do segundo ao quinto segmento, e pela presença de 16 setígeros torácicos. Com base nesse presumido monofiletismo, foi realizada uma análise cladística para tentar determinar as relações filogenéticas entre as espécies conhecidas. A análise dos dados gerou 14 arvores mais parcimoniosas, cujo consenso permitiu o reconhecimento da seguinte relação filogenética $(O$. sexlobatus $(O$. floriceps $(O$. antarcticus (O. pacificus (O. phyllocomus ((O. japonicus, O. lingulatus) (O. longipes, O. myunus $))))))$ ).
\end{abstract}

Palavras chave: Polychaeta, Trichobranchidade, Octobranchus, Análise Cladística

\section{CLADISTIC ANALYSIS OF OCTOBRANCHUS MARION \& BOBRETZKY, 1875 (TRICHOBRANCHIDAE: POLYCHAETA)}

\begin{abstract}
The genus Octobranchus contains nine valid species: Octobranchus pacificus (Berkeley \& Berkeley, 1954), O. antarcticus Monro, 1936, O. floriceps Kingston \& Mackie, 1980, O. japonicus Hessle, 1917, O. lingulatus (Grube, 1863), O. longipes Blankensteyn \& Lana, 1987, O. myunus Hutchings \& Peart, 2000, O. phyllocomus Hartman, 1952 and O. sexlobatus Hartmann- Schröder \& Rosenfeldt, 1989 with a wide world distribution (North Sea, Sea of Japan, Australia, Brazil, Mediterranean and Antarctica). The monophyly of Octobranchus is sustained by the presence of four pairs of gills from the second to the fifth segments, and for the presence of 16 thoracic setigers. Based in this presumed monophyly, a cladistic analysis was done to determine the phylogenetic relationships among the species. The analysis of the data generated 14 most parsimonious trees, whose consense allowed to recognize the following phylogenetic relationship (O. sexlobatus (O. floriceps (O. antarcticus $(O$. pacificus $(O$. phyllocomus $((O)$ japonicus, $O$. lingulatus) (O. longipes, O. myunus))))))).
\end{abstract}

Key words: Polychaeta, Trichobranchidade, Octobranchus, cladistic analysis 


\section{INTRODUÇÃO}

O gênero Octobranchus é caracterizado, entre os Trichobranchidae, pela presença de quatro pares de brânquias filiformes, foliáceas ou em forma de roseta, do segundo ao quinto segmentos. Manchas ocelares e lamelas laterais estão presentes nos segmentos anteriores. As notosetas, sempre lisas, estão presentes em 16 setígeros torácicos, contados a partir do segmento 4 e as neurosetas, desde o segmento 7 (setígero 4) até o pigídio. As neurosetas torácicas são manúbrio-aviculares e as abdominais, breviaviculares.

Atualmente o gênero possui nove espécies válidas: Octobranchus pacificus (Berkeley \& Berkeley, 1954), O. antarcticus Monro, 1936, O. floriceps Kingston \& Mackie, 1980, O. japonicus Hessle, 1917, O. lingulatus (Grube, 1863), O. longipes Blankensteyn \& Lana, 1987, O. myunus Hutchings \& Peart, 2000, O. phyllocomus Hartman, 1952 e O. sexlobatus Hartmann-Schröder \& Rosenfeldt, 1989. As espécies do gênero possuem uma ampla distribuição, podendo ser encontradas no Mar do Japão, na Austrália, no Brasil, no Mar do Norte, no Mediterrâneo e na Antártica.

São poucos os problemas taxonômicos atuais do gênero. Sua espécie-tipo foi originalmente descrita por Grube (1863) sob o nome de Terebella lingulatus e posteriormente referida a Octobranchus, tal como definido por Marion \& Bobretzky (1875). O gênero Novobranchus, criado para a espécie Novobranchus pacificus Berkeley \& Berkeley, 1954 , foi mais recentemente referido a Octobranchus por Kingston \& Mackie (1980), com base no número de brânquias e de setígeros torácicos.

Com o objetivo de investigar a relação filogenética das espécies do gênero e assim poder sugerir o melhor argumento para sua classificação, foi realizada uma análise cladística de Octobranchus. Esse trabalho faz parte de um projeto maior que visa a análise cladística de toda a família Trichobranchidae, que até o momento não havia sido objeto de qualquer estudo filogenético.

\section{MATERIAIS E MÉTODOS}

Para a construção da matriz de caracteres foi utilizada a literatura primária de Trichobranchidae, incluindo as descrições originais de todas as espécies referidas ao gênero, complementadas por revisões ou sinopses recentes (Kingston \& Mackie, 1980; Blankensteyn \& Lana, 1987). A análise bibliográfica foi reforçada ou completada com o exame de material de referência ou parátipos de 4 espécies, disponíveis no Centro de Estudos do Mar da Universidade Federal do Paraná, ou por empréstimo do Museu da Austrália e no Museu de Hamburgo, Alemanha (Tabela 1).

O grupo foi considerado monofilético a priori, embora esta presumida monofilia tenha sido criticamente testada no decorrer da análise. Todos os nove taxóns nominais de Octobranchus foram utilizados como terminais. Evidências para a monofilia de cada terminal do grupo são também apresentadas como autapomorfias na análise. Na reconstrução das relações filogenéticas, foi adotado o método de comparação holomorfológica (Hennig, 1966; Wiley, 1981; Amorim, 1997) na elaboração das hipóteses sobre direcionalidade dos dendrogramas produzidos. Utilizou-se também o programa Hennig86 versão 1.5 (Farris, 1989) através de seu comando de algoritmo exato, ie, na tentativa de se encontrar o cladograma mais parcimonioso. Também utilizou-se o aplicativo Tree Gardener v.2.2 (Ramos, 1996), que possibilita uma melhor visualização gráfica dos cladogramas encontrados, os comandos de consenso estrito criado por Sokal \& Rohlf (1981) e a pesagem sucessiva desenvolvido por Farris (1969).

Na polarização dos caracteres foi utilizado o método de comparação por grupos externos múltiplos (Nixon \& Carpenter, 1993; Amorim, 1997). O grupo externo utilizado para 
realizar a análise foi composto por três espécies de gêneros próximos, pertencentes à mesma família, Trichobranchus bibranchiatus Moore, 1903, Terebellides lobatus Hartman \& Fauchald, 1971 e Terebellides anguicomus, Müller 1858.

Todos os onze caracteres (Tabela 2) utilizados para a montagem da matriz (Tabela
3) foram codificados como binários, seguindo a orientação de Pleijel (1995). Não foi possível obter informações a respeito de todos os caracteres para todos os taxa examinados. Nestes casos, adotou-se o código de "ausência de informação" para composição da matriz.

Tabela 1. Lista do material examinado, com o local de depósito e referências consultadas

\begin{tabular}{|c|c|}
\hline Espécie & Museus e referências \\
\hline Octobranchus antarcticus & Monro, 1936; Kingston \& Mackie, 1980 \\
\hline Octobranchus lingulatus & Moore, 1903; Hutchings \& Peart, 2000 \\
\hline Octobranchus longipes & $\begin{array}{l}\text { Museu do Centro de Estudos do Mar- BPO-247 } \\
\text { (Holótipo, } 1 \text { espécime) }\end{array}$ \\
\hline Octobranchus myunus & $\begin{array}{l}\text { Museu da Austrália - W24183 } \\
\text { espécime) }\end{array}$ \\
\hline Octobranchus pacificus & $\begin{array}{l}\text { Berkeley \& Berkeley, 1954; Kingston \& Mackie, } \\
1980\end{array}$ \\
\hline Octobranchus phyllocomus & Hartman, 1952; Hutchings \& Peart, 2000 \\
\hline Octobranchus sexlobatus & Museu de Zoologia de Hamburgo - (1 espécime) \\
\hline Terebellides anguicomus & $\begin{array}{l}\text { Museu do Centro de Estudos do Mar- BPO-333 } \\
\text { (9 espécimens) }\end{array}$ \\
\hline ellides lobatus & Hartman \& Fauchald, 1971 \\
\hline Trichobranchus bibranchiatus & Moore, 1903; Imajima \& Williams, 1985 \\
\hline
\end{tabular}

Tabela 2. Sumário da lista de caracteres de Octobranchus.

1. Quatro pares de brânquias: 0- ausente; 1- presente

2. Brânquias basalmente grossas e afilando-se posteriormente: 0- ausente; 1presente

3. Dezesseis setígeros torácicos: 0 - ausente; 1 - presente

4. Tentáculos: 0 - 2 tipos; 1 - 1 tipo

5. Uncini abdominal brevi avicular: 0- 1 dente principal e 2 fileiras de dentes secundários; 1 - 1 dente principal e 3 fileiras de dentes secundários

6. Primeiro notopódio menos desenvolvido que os demais: 0 - ausente; 1 - presente

7. Lamelas laterais: 0 - ausente; 1 - presente

8. Lamelas laterais do segmento 1 ao 4 : 0 - ausente; 1 - presente

9. Neurosetas: 0 - originadas no segmento $6 ; 1$ - originadas no segmento 7

10. Ocelos: 0- ausente; 1 - presente

11. Papilas nefridiais: 0 - ausente; 1 - presente 


\section{RESULTADOS E DISCUSSÃO}

Foram obtidas 14 árvores mais parcimoniosas com um comprimento de 21 passos, índice de consistência igual a 52 e índice de retenção igual a 69 . Posteriormente foi utilizado o consenso estrito e pesagem sucessiva para obtenção de apenas uma árvore mais parcimoniosa (Figura 1).

O cladograma final encontrado está bem resolvido, sem nenhuma politomia no grupo interno. A leitura dessa única árvore ótima fornece elementos que efetivamente demonstram que o gênero Octobranchus é monofilético. Tal afirmação se baseia inicialmente nos caracteres 1 e 3 , ou seja, na presença de 4 pares de brânquias e 16 setígeros torácicos, que formam sinapomorfias compartilhadas por todos os integrantes do gênero. Além disso, os caracteres 8 e 9 , ou seja, lamelas laterais do segmento 1 ao 4 e neurosetas originadas no segmento 7 , aparecem também como sinapomorfias do grupo interno, sofrendo, po- rém, reversões secundárias. A reversão do caráter 8 acontece independentemente nos táxons Octobranchus antarcticus e no clado (O. longipes + O. myunus), sendo esta segunda reversão uma sinapormorfia para este grupo, pois Octobranchus longipes possui as lamelas branquiais nos segmentos 2 ao $6 \mathrm{e}$ Octobranchus myunus nos segmentos 2 ao 4 . Já o caráter 9 também sofreu reversões independentes nos táxons Octobranchus pacificus e O. phyllocomus.

O aparecimento do caráter 11, papilas nefridiais, é uma sinapomorfia para o agrupamento monofilético (Octobranchus pacificus + $O$. antarcticus $+O$. japonicus $+O$. lingulatus + O. longipes + O. myunus + O phyllocomus) e sua perda (reversão) é uma sinapomorfia mais inclusiva que define o agrupamento ((Octobranchus japonicus) + (O. lingulatus $)$ ) .

O caráter 2 , brânquias basalmente grossas e afilando-se posteriormente, define o agrupamento monofilético ((Octobranchus phyllocomus $)+(0$. japonicus $)+(O$. lingulatus $)$

Tabela 3. Matriz de dados usada na análise cladística do gênero Octobranchus; $0=$ ausente; $1=$ presente; ? = ausência de informação; - = dado inaplicável.

\begin{tabular}{|c|c|c|c|c|c|c|c|c|c|c|c|}
\hline Taxa & \multicolumn{11}{|c|}{ Caracteres } \\
\hline & 1 & 2 & 3 & 4 & 5 & 6 & 7 & 8 & 9 & $\begin{array}{l}1 \\
0\end{array}$ & $\begin{array}{l}1 \\
1\end{array}$ \\
\hline $\begin{array}{l}\text { Grupo Externo } \\
\text { Terebellides lobatus }\end{array}$ & 0 & 0 & 0 & 0 & 0 & 0 & 1 & 0 & 0 & 0 & 0 \\
\hline Terebellides anguicomus & 0 & 0 & 0 & 0 & 0 & 0 & 1 & $?$ & 0 & 0 & $?$ \\
\hline $\begin{array}{c}\text { Trichobranchus bibranchiatus } \\
\text { Grupo Interno }\end{array}$ & 0 & 0 & 0 & 1 & 1 & 1 & 0 & - & $?$ & 0 & 0 \\
\hline Octobranchus antarcticus & 1 & 0 & 1 & 0 & 0 & 1 & 1 & 0 & 1 & 1 & 1 \\
\hline Octobranchus floriceps & 1 & 0 & 1 & 0 & 0 & 1 & 1 & 1 & 1 & 1 & 0 \\
\hline Octobranchus japonicus & 1 & 1 & 1 & 1 & 1 & 1 & 1 & 1 & 1 & ? & 0 \\
\hline Octobranchus lingulatus & 1 & 1 & 1 & 1 & 1 & 0 & 1 & 1 & 1 & 1 & 0 \\
\hline Octobranchus longipes & 1 & 1 & 1 & 1 & 1 & 1 & 1 & 0 & 1 & 1 & 1 \\
\hline Octobranchus myunus & 1 & 1 & 1 & 1 & 1 & 1 & 1 & 0 & 1 & 1 & 1 \\
\hline Octobranchus pacificus & 1 & 0 & 1 & 1 & 0 & ? & 1 & ? & 0 & 1 & 1 \\
\hline Octobranchus phyllocomus & 1 & 1 & 1 & 1 & 0 & 1 & 1 & 1 & 0 & 1 & 1 \\
\hline Octobranchus sexlobatus & 1 & 0 & 1 & 0 & 1 & 0 & 1 & 1 & 1 & 0 & 0 \\
\hline
\end{tabular}




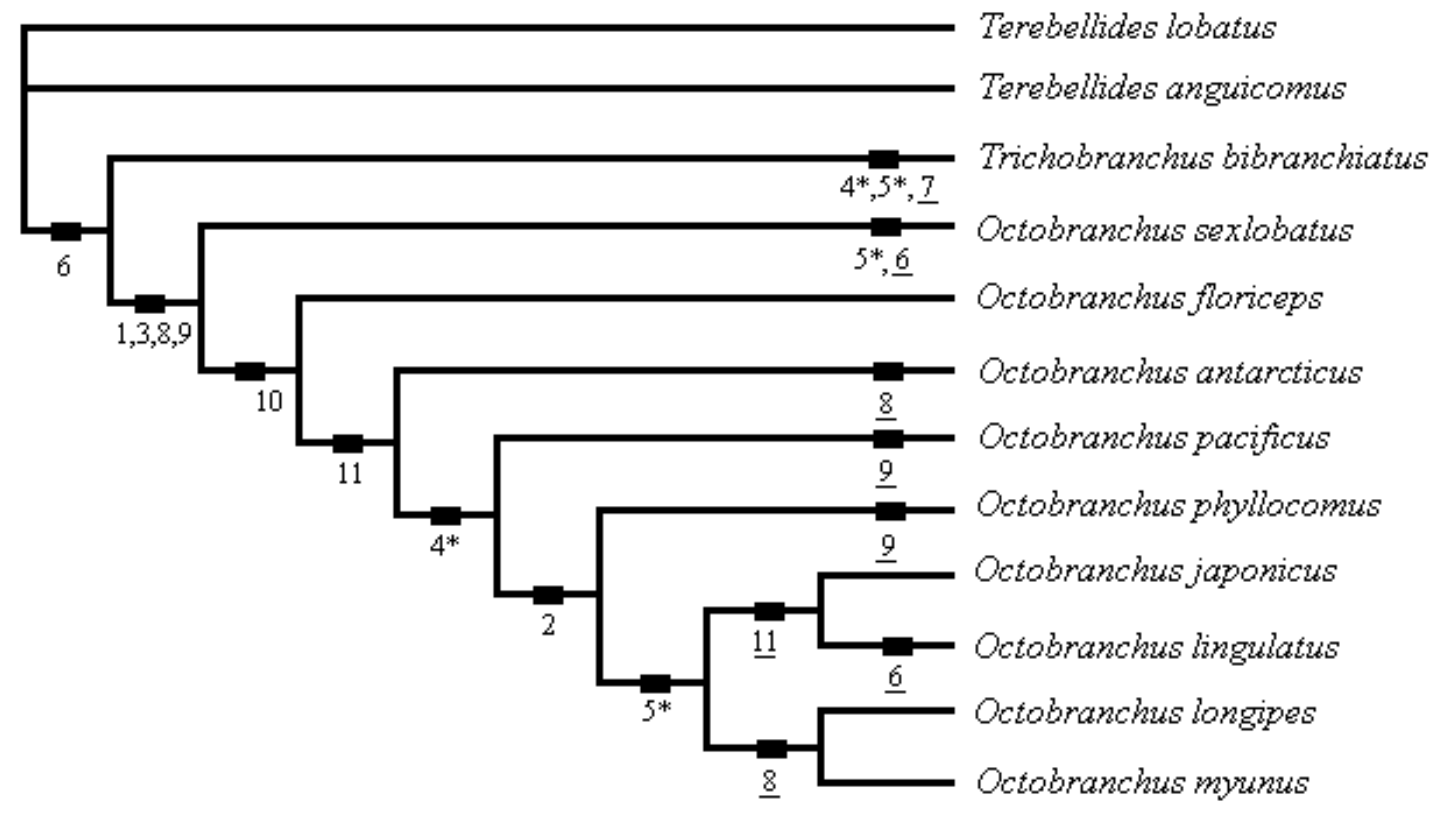

Figura 1. Cladograma final com as relações entre as espécies do gênero Trichobranchus, obtida através do uso do algoritmo exato ie, consenso estrito e pesagem sucessiva, obtendo uma árvore de c 77, ci 19 e ri 89. Números com asteriscos representam homoplasias, números sublinhados representam reversões e os números em arábico representam as sinapomorfias.

$+($ O. longipes $)+($ O. myunus $))$, e o caráter 5 , uncini abdominal brevi avicular com um 1 dente principal e 3 fileiras de dentes secundários, define por sua vez o grupo $((O$. japonicus $)+$ (O. lingulatus $)+($ O. longipes $)+(0$. myunus $))$. Porém, esse último caráter aparece independentemente no táxon Trichobranchus bibranchiatus, pertencente ao grupo externo.

A ausência de politomias no cladograma de consenso e pesagem é um claro indicativo da existência de um grupo de sinapomorfias que sustentam todos os clados. As sinapomorfias "número de brânquias e número de setígeros torácicos" efetivamente diagnosticam o gênero Octobranchus, já que os caracteres "presença de lamelas laterais e "local de origem das neurosetas" sofrem reversão, estando ausentes em alguns táxons terminais.
Apesar da definição de uma série de clados diagnosticados por sinapomorfias inclusivas ao gênero, não se justifica a criação de novas categorias lineanas no nível de sub-gênero, já que isto acarretaria a parafilia dos novos grupos. A análise mostra que foi essencialmente correto o procedimento de Mackie \& Kingston (1980), ao referirem Novobranchus a Octobranchus.

Em síntese, a presente análise demostra conclusivamente a monofilia de Octobranchus, validando a categorização taxonomica atualmente adotada para o clado.

\section{AGRADECIMENTOS}

A Penny Berents e Angelika Brandt, curadoras do Museu da Austrália e do Museu de Zoologia de Hamburgo, pelo empréstimo 
de material de suas coleções e a Ana Claudia dos Santos Brasil pelos comentários efetuados durante a redação desse trabalho.

\section{REFERÊNCIAS BIBLIOGRÁFICAS}

Amorim, D.S. 1997. Elementos básicos de sistemática filogenética. $2^{a}$ ed. Holos Editora.

Berkeley, E. \& C. Berkeley. 1954. Additions to the polychaeta fauna of Canada, with comments on some older records. J. Fish. Res. Bd Can. 11: 454-471.

Blankensteyn, A. \& P.C. Lana. 1987. Octobranchus longipes sp. $\mathrm{n}$. (Trichobranchidae; Polychaeta) da costa sudeste do Brasil. Arq. Biol. Technol. 30: 671-676.

Farris, J.S. 1969. A successive approximations approach to character weighting. Syst. Zool. 18: 374-385.

Farris, J.S. 1989. The retention index and the rescaled consistency index. Cladistics. 5: 417-419.

Grube, A.E. 1863. Beschreibung neuer oder wenig bekannter Anneliden. Arch. Naturgesch. Berlin. 29: 37-69, pl.IV-VI.

Hartman, O. 1952. The marine annelids of the United States Navy Antarctic Expedition, 1947-48. J. Wash. Acad. Sci. 51: 9-20.

Hennig, W. 1966. Phylogenetic systematics. Univ. Illinois Press, Urbana.

Hutchings, P.A \& R. Peart. 2000. A revision of the Australian Trichobranchidae. Invert. Taxon. 14: 225-272

Imajima, M. \& S.J. Williams. 1985. Trichobranchidae (Polychaeta) chiefly from the Sagami and Suruga Bays, collected by R/v Tansei Maru (Cruises KT-65-76). Bull. Natn. Sci. Mus., Ser. A. 11(1): 7-18

Kingston, P.F. \& A.S.Y. Mackie. 1980. Octobranchus floriceps sp. nov. (Polychaeta: Trichobranchidae) from the northern North Sea with a re-examination of O. antarcticus Monro. Sarsia. 65: 249254
Marion, A.F. \& N. Bobretzky. 1875. Etude des Annélides du Golfe de Marseille. Ann Sci. Nat. Paris. (6)2: 1-106

Monro, C.C. 1936. Polychaete worms. II. Discovery Rep. 12: 59-198

Moore, P. 1903. Polychaeta from the coastal slope of Japan and from Kamchatka and Bering Sea. Proc. Acad. nat. Sci. Philad. 55: 401-490.

Nixon, C.K. \& J.M. Carpenter. 1993. On Outgroups. Cladistics. 9:413-426.

Pleijel, F. 1995. On character coding for phylogeny reconstruction. Cladistics. 11: 309-315

Ramos, T.C. 1996. Tree Gardener version 2.2. P.C. program for windows

Sohal, R.R. \& F.J. Rohlf. 1981. Taxononic congruence in the Leptopomorpha reexamined. Syst. Zoo. 30, 309-325

Wiley, E.O. 1981. Phylogenetics: the theory and practice of phylogenetic systematics. John Wiley and Sons, New York. 